by researchers at the Universities of Helsinki and Oulu, Finland. Sleep quality was measured using actigraphs, and the Sleep Disturbance Scale for Children and the ADHD Rating Scale IV were administered to parents. Of 280 children (134 boys, 146 girls) with a mean age of 8.1 years (range 7.4-8.8), those with a short average sleep duration $(<7.7$ hours) had higher hyperactivity/impulsivity and ADHD total scores, but similar inattention scores compared with children sleeping 7.7 to 9.4 hours or $>9.4$ hours. Short sleep duration remained significantly associated with hyperactivity/impulsivity when controlling for basic confounding variables and also for sleeping difficulties and somatic illnesses, but it was not related to inattention or the ADHD total score. Short sleep duration was not correlated with sleeping difficulties, but sleep-breathing disorder was significantly associated with hyperactivity/impulsivity, inattention, and ADHD total score. Parent-reported short sleep duration was not related to hyperactivity/impulsivity, inattention, and the ADHD total score. (Paavonen EJ, Raikkonen K, Lahti J, et al. Short sleep duration and behavioral symptoms of attention-deficit/hyperactivity disorder in healthy 7- to 8-year-old children. Pediatrics May 2009;123:e857-e864). (Respond: Dr Paavonen. E-mail: juulia.paavonen@,helsinki.fi).

COMMENT. Short sleep duration measured objectively with the actigraph and parent-reported sleeping difficulties are independently associated with increased risk of behavioral symptoms of ADHD. One third of children in the US are estimated to have inadequate sleep (Smaldone A et al. Pediatrics 2007;119(suppl 1):S29-S37). Questions regarding sleeping habits are important in the evaluation of children with ADHD, especially in relation to the symptoms hyperactivity/impulsivity and their association with short sleep duration or sleeping difficulties, such as sleep-breathing disorder and snoring. Short sleep duration is not correlated with the symptoms of inattention. A causal relation between sleep duration and behavioral symptoms is not established, but maintaining regular sleeping schedules may help to ameliorate the hyperactivity and impulsivity of ADHD. Sleep duration and sleeping difficulty studies are often inaccurate, relying heavily on parental reports, which are susceptible to bias. In a child with hyperactive behavior, excessive snoring should prompt referral to ENT, and sleeping difficulties may indicate the need for a polysomnograph. However, polysomnographic sleep scores are not related to academic functioning, IQ and neuropsychological test cores are powerful predictors of achievement. (Mayes SD et al. 2008). Inattentive symptoms are sometimes related to daytime sleepiness. (Willoughby MT et al. 2008).

Girls with ADHD generally have a greater frequency of the inattentive subtype than boys, but overall, boys outnumber girls with a 4:1 ratio for the ADHD-HI and 2:1 for ADHD-AD. (Wolraich ML et al. 1996). The preponderance of girls in the above study is unusual, but apparently, gender was not a modifying factor.

\title{
STRUCTURAL CEREBRAL CHANGES AND CONDUCT DISORDER
}

Voxel-based morphometry was used to analyze structural MRI scans from 23 boys with callous-unemotional conduct problems (mean age 11 yrs 8 months) and 25 healthy controls, in a study at the Institute of Psychiatry, King's College, London, UK, and other centers in London and Germany. Both grey matter volume and concentration were examined, controlling for cognitive ability and ADHD. Boys with unemotional conduct problems showed increased grey matter concentration in the medial orbito-frontal and anterior 
cingulate cortices, and increased grey matter volume and concentration in the temporal lobes bilaterally and the cerebellum. The findings were consistent with a delay in cortical maturation in cortical areas involved in decision making, morality and empathy in boys with callous-unemotional conduct problems. (De Brito SA, Mechelli A, Wilke M, et al. Size matters: increased grey matter in boys with conduct problems and callous-unemotional traits. Brain April 2009;132:843-852). (Respond: Dr Stephane A De Brito, Institute of Psychiatry, PO Box 23, King's College, London, UK. E-mail: stephane.debrito@iop.kcl.ac.uk).

COMMENT. Contrary to previous reports of decreased concentration and volume of cortical areas in psychopathic adolescents and adults with conduct disorders, this study found increases in grey matter concentration in orbito-frontal cortex and anterior cingulate cortex in 11-year-old patients. Blair RJR, of the National Institute of Mental Health, Bethesda, MD, in an editorial, remarks on the advances in the science of a neural substrate for lack of emotion, and in driving callous behavior in boys with conduct problems. The progress is not only in tolerance for the field of neurobiology of anti-social behavior but also its sophistication. (Brain 2009;132:831-832). Children with callous-unemotional traits have an increased risk for anti-social behavior. A better understanding of the cause should lessen the stigma and facilitate appropriate treatment. Children with ADHD complicated by unemotional conduct disorders should receive psychiatric care to lessen the risk of anti-social behavior.

\section{MOVEMENT DISORDERS}

\section{CLONIDINE AND LEVETIRACETAM FOR TICS COMPARED}

The efficacy of clonidine and levetiracetam for treating tics in Tourette syndrome was compared in a 15-week randomized, double-blind, flexible dose, crossover study at Johns Hopkins Hospital, Baltimore, MD. In 10 subjects, ages 8-27 years, the mean Total Tic Score improved significantly with clonidine compared with levetiracetam, whereas the Yale Tic Severity Scale did not change. With levetiracetam, none of the measures showed a change. The initial dose of clonidine was $0.05 \mathrm{mg}$, twice daily, and levetiracetam $10 \mathrm{mg} / \mathrm{kg} / \mathrm{day}$, divided twice daily. The mean maximum dose for clonidine was $0.20 \mathrm{mg} / \mathrm{day}$, and for levetiractam, $1,150 \mathrm{mg} / \mathrm{day}$. The mean Tic Score and Tic Severity Scale scores at baseline were similar in treatment groups. Sedation occurred with clonidine in 5 patients and irritability as a side effect of levetiracetam in 4 . Clonidine reduced the frequency of tics but levetiracetam had no effect. (Hedderick EF, Morris CM, Singer HS. Double-blind, crossover study of clonidine and levetiracetam in Tourette syndrome. Pediatr Neurol 2009;40:420425). (Respond: Dr Hedderick, Johns Hopkins Hospital, Suite 2158, 200 N Wolfe Street, Baltimore, MD 21231. E-mail: ehedder1@jhmi.edu).

COMMENT. Although this study found a statistically significant improvement in Total Tic Score with clonidine, the authors considered the benefit of limited clinical significance. Previous reports demonstrate a range of results with clonidine, from 0 to $75 \%$ reduction in tics (authors reference). As the authors comment, additional direct comparison studies, including placebo, in a larger series would be more meaningful. 